\title{
Research on IRP of Perishable Products Based on Mobile Data Sharing Environment
}

\author{
Zelin Wang, School of Information Science and Technology, Nantong University, Nantong, China \\ Xiaoning Wei, School of Information Science and Technology, Nantong University, Nantong, China \\ Jiansheng Pan, School of Information Science and Technology, Nantong University, Nantong, China
}

\begin{abstract}
Inventory routing problem (IRP) has always been a hot issue. Due to its particularity, perishable products have high requirements for inventory and transportation. In order to reduce the losses of perishable goods and improve the storage efficiency of perishable goods, based on the general inventory path problem, this paper further has studied the IRP of perishable goods. In addition, in the process of product distribution and transportation, there are a lot of real-time product information generated dynamically. These real-time mobile data must be shared by the whole distribution network, which will also dynamically affect the efficiency of IRP research. On the basis of some assumptions, the mathematical model has been established with inventory and vehicle as constraints and the total cost of the system as the objective. In view of the particularity of perishable inventory path problem, this paper proposed an improved differential evolution algorithm (IDE) to improve the differential evolution algorithm from two aspects. Firstly, the population has been initialized by gridding and the greedy local optimization algorithm has been used to assist the differential evolution algorithm, with these measures to improve the convergence speed of the algorithm. Then, the accuracy of the algorithm is improved by the adaptive scaling factor, two evolution modes and changing the constraints of the problem. Then the improved algorithm has been used to solve the inventory path problem. The results of numerical experiments show that the algorithm is effective and feasible and can improve the accuracy and speed up the convergence of the algorithm.
\end{abstract}

\section{KEYWORDS}

Accuracy of the Algorithm, Convergence Speed of the Algorithm, Differential Evolution Algorithm, Inventory Routing Problem

\section{INTRODUCTION}

Inventory-routing problem (IRP) is the key to determine the inventory strategy and distribution strategy. The purpose of inventory strategy is to determine the distribution target and quantity of goods for each planning period, and distribution solves the centralized scale effect of logistics and the scattered demand of customers, and the purpose of distribution strategy is to determine the distribution route of goods. IRP tries to minimize the sum of inventory costs and distribution costs. IRP is an integration of inventory issues and distribution issues that needs to be addressed on the same platform at the same time. Because these two questions are opposite and contradictory to each other, in the pursuit of the minimum inventory cost, it will inevitably bring the maximum distribution cost; on the contrary, if the pursuit of the minimum distribution cost, it will inevitably bring the maximum inventory cost. But at the same time, solving these two problems is a very difficult event. Both of them are N-P difficult

DOI: $10.4018 /$ IJCINI.20210401.oa10

This article published as an Open Access article distributed under the terms of the Creative Commons Attribution License (http://creativecommons.org/licenses/by/4.0/) which permits unrestricted use, distribution, and production in any medium, provided the author of the original work and original publication source are properly credited. 
problems. Especially when the number of customers in distribution is large and the demand of each customer is random, the optimal strategy of IRP problem is often very complex, and the solution of the problem often makes the quantity of distribution and the distribution room. Distribution routes lack stability. The value and freshness of perishable products are highly correlated, which is a special population of IRP. Due to the prolongation of perishable products over time, the freshness of products will decrease and the deterioration degree will increase. Therefore, the timeliness, temperature control and other preventive measures of perishable products distribution require higher requirements, resulting in higher distribution costs. As a result, the goods will be seriously damaged and depreciated in value. Therefore, it is more urgent and realistic to study the IRP of perishable products.

In addition, in the process of distribution and transportation, there are many mobile dynamic data, which must be shared by all nodes in time to update the distribution inventory plan in time. Therefore, the research data and the research object cannot be separated from the dynamic environment. Moreover, terminal logistics has the characteristics of service area distribution, various media and weak value added, so it often leads to poor information communication. How to improve information sharing, and how to improve the level of distribution service in such an environment, become the problems. In this paper, an improved differential evolution algorithm is used to find the approximate optimal solution of the IRP problem for perishable products.

In the available literature, (Yu Li, Shuhua Zhang and Jingwen Han, 2017) studies an inventory level model that (Samira Mirzaei and Abbas Seifi, 2015) established an inventory path optimization model based on freight cost, inventory cost and sales loss cost. Combined with simulated annealing and tabu search, he designed a meta heuristic algorithm. (Bhattacharjee, et al., 2007) established a multi-period ordering and pricing model, and solved a deteriorating product with a fixed period by using two different heuristic algorithms. (Levin Y, 2006) and others assume that the demand of perishable products obeys Poisson distribution, and that the retailer's dynamic pricing strategy under the condition that demand will be affected by price. (Goyal and Giri, 2001) firstly reviewed the inventory status of perishable goods. During this period, the research on supply chain theory reached a new height.

(Donselaar M, V,Woensel, T.V and Broekmeulen R, 2006) Through theoretical analysis, it is proved that the sum of inventory cost and distribution cost under the optimal strategy of partitioning customers is $98.5 \%$ optimal. Zoning can effectively simplify the problem and reduce the difficulty of the problem, so this paper also uses the idea of fixed zoning, and does not need to spend a lot of cost for low probability events. (Alvareza A, Jean-Franc, Ois Cordeaub and Jansb R, 2019) proposed Branch-and-cut algorithms and hybrid heuristic solution method to solve inventory routing problem in which goods are perishable. (Herbon A and Devapriya P, 2017)studied the inventory distribution optimization of fresh products from the perspective of supply chain operation management. (Wenhui Zhang, Qiqi Miao, Feng Guan, et al. 2018)Based on the time window of perishable products acceptable to customers, a mathematical model was established aiming at the lowest distribution cost. The distribution area was divided by scanning algorithm in advance, and the optimal path was solved by genetic algorithm. (Ming, wang yong and YH Li. 2018) designed decision variables based on a customer's seven requirement attributes,and proposed a fuzzy clustering method for grouping customers and improved a fuzzy genetic algorithm that was used to solve the proposed total cost model.

In solving IRP problems, because of the complexity of the problem itself, when the scale of the problem itself is large, it is a very difficult event to find the optimal solution. (Rafie-Majd Z, Pasandideh S H R and Naderi B, 2017) tried to solve IRP problems by using heuristic variable neighbor search intelligent method. IRP is solved in two stages. Firstly, heuristic variable neighbor search is adopted. In this stage, the inventory cost is not considered. The purpose is to obtain a feasible initial solution. Then the second stage iteratively optimizes the initial solution and achieves very good results. (Mjirda A, Jarboui B, Macedo R and Hanafi S., 2017) tried to use heuristic tabu search intelligent algorithm to solve the shortest inventory path problem, and compares the operation effect of the algorithm with that of the original Lagrange relaxation algorithm, which proves that this method has obvious superiority. 
(Wouter L, El-Houssaine A, Khaled H H, et al., 2018)developed new sets of valid inequalities to strengthen the linear relaxation, and improved the coefficients in the inventory management constraints, and presented a stronger formulation for the routing component of the problem, and proved that a large number of variables can be eliminated during a preprocessing stage. (Darestani S A, Mousazadeh S, 2019) studied two questions of robust and flexible for the production, inventory and routing blood products. They solved by heuristic (local search) and meta-heuristic (Adaptive Large Neighborhood Search (ALNS)) algorithms, which are the methods of choice in particular for NP-hard problems. (Jafarkhan, Fatemeh, Yaghoubi, et al., 2018) analyzed flexible and robust inventory-routing where a blood center distributes the blood cell to hospitals under uncertain demand and supply. (Alvarez, Aldair; Cordeau, Jean-Francois; Jans, Raf., 2020)studied an inventory routing problem in which goods are perishable, and introduced four mathematical formulations for the problem, two with a vehicle index and two without a vehicle index, and proposed branch-and-cut algorithms to solve them, and proposed a hybrid heuristic based on the combination of an iterated local search meta-heuristic and two mathematical programming components.

From (Kangshun Li, et al., 2018, 2019), it can be seen that in recent years, differential evolution algorithm has achieved remarkable results in solving large-scale combinatorial optimization problems and function optimization problems because of its global convergence and robustness. In this paper, an improved differential evolution algorithm is proposed to deal with the IRP problem of perishable products.

\section{PROBLEM MODEL}

\subsection{Study on Freshness of Perishable Products}

The freshness of perishable commodities is the key point of this paper. In the study of freshness loss of perishable products, scholars mainly focus on the change of freshness of perishable products with time, often with the time, the freshness decreases. While the research on the relationship between freshness of fresh products and transportation distance is relatively small.

In the study of the freshness of perishable products changing with time, freshness is generally regarded as a continuous decreasing function with time. The function is generally expressed as follows:

$$
\theta(t)=\frac{1}{1+\alpha t^{2}}
$$

where $a>0$ indicates the sensitivity of freshness of perishable products to time. For the freshness function of perishable products, if we take the derivative of time $t$, we can get

$$
\frac{d \theta(t)}{d t}=\frac{-2 \alpha t}{\left(1+\alpha t^{2}\right)^{2}} \leq 0
$$

From the first derivative, we can see that freshness is a continuous decreasing function of time, that is, with the passage of time, the freshness of perishable products will gradually decline. The freshness of perishable products in this paper is only related to the distance. The longer the transportation distance is, the freshness decreases. That is, freshness is also a continuous decreasing function of the distribution route. We assume that there is a certain functional relationship between freshness loss and distance. After statistical analysis and functional fitting of the relevant data, we get that the function in the relevant definition domain is an exponential function, that is, 


$$
\psi=\alpha \theta^{k d}, \frac{d_{\psi}}{d_{d}}=\alpha k \theta^{k d}
$$

Among them, $a$ and $k$ are constants greater than $0,0<=\psi<=1$, d denotes the distance of perishable products from suppliers to retailers. Then $\psi$ is the freshness of perishable products.

\subsection{Model Construction}

\subsubsection{Model Hypothesis}

Before the model is finally established, several assumptions need to be made about the model:

(1) Retailers' demand for goods is related to freshness and price, the demand of retailers is random and independent, and obeys normal distribution.

(2) Within a delivery period, each vehicle can provide distribution services to multiple retailers, but only one vehicle can serve one retailer, and each vehicle only travels once for delivery.

(3) For inventory, the retailer's inventory cost is considered only, without considering the supplier's inventory cost and inventory quantity.

(4) .The supplier carries on the transportation of a perishable product to the retailer by only road style.

(5) The supplier managed inventory (VMI) model is adopted, and the supplier can control the retailer's inventory through monitoring.

(6) Retailers adopt (S, R, T) inventory strategy, and there is no replenishment lead time problem.

(7) The supplier has enough delivery vehicles and the distribution vehicles are identical, and the vehicle load is limited.

(8) There is a supplier, many retailers, and the location coordinates of these businesses are determined. In order to reflect the problem more clearly and intuitively, the transportation distance only considers the straight line distance between the points.

(9) No consideration is given to the service time of the vehicle at the customer's office, i.e. instantaneous completion;

(10) During transportation, the freshness of perishable products will change, but it is only related to the transportation distance.

\subsubsection{Relevant parameters and symbolic descriptions}

(1) Relevant parameters

$Q_{i}$ : Retailer i's maximum inventory.

$t$ : cycle time.

$M$ : Number of distribution vehicles.

$N$ : Number of retailers.

$W$ : Load limits for distribution vehicles.

$U_{i}$ : Retailer i's inventory upper limit.

$L_{i}$ : Retailer i's inventory lower limit.

$D_{i j}$ : the distance between retailer $i$ and retailer $j, i=0$ means supplier;

$\Psi_{i}:$ Freshness loss of perishable product $i$;

$P_{v}:$ the original price per unit product;

$P_{i}$ : unit out-of-stock cost of retailer $i$;

$C_{0}$ : Fixed start-up costs incurred by suppliers per delivery;

$C_{l}$ : unit transportation cost of vehicle transportation;

$h_{i}$ : Retailer $i$ unit products do not consider freshness reduction in storage costs; 
$a_{i}$ : the level of service that retailer $i$ should provide to consumers is related to the minimum inventory level.

(2) Variables

$X_{t i}:$ The demand of retailer $i$ in the $t$-cycle conforms to the normal distribution $N\left(\mu, \sigma^{\wedge} 2\right)$, and the distribution function is $F_{i}(x)$.

$S_{t i}:$ Initial inventory of retailer $i$ in $t$ cycle;

$Q_{t i}:$ Supplier's replenishment to retailer $i$ at the end of the $t$-cycle;

$x_{M t i j}=\left\{\begin{array}{cc}1 & \text { attheendof cyclet } \\ & \text { vehicle } M \text { drives from retailerito } j \\ 0 & \text { othersize }\end{array}\right.$

$$
Y_{M t i}=\left\{\begin{array}{cc}
1 & \text { attheendof cyclet } \\
& \text { vehicle M servicve for } i \\
0 & \text { othersize }
\end{array}\right.
$$

\subsection{Cost Analysis}

The secondary supply chain system established in this paper is a typical R-System. R-System is based on the assumption that all the demanders in the supply chain system should be managed by a central organizer or decision maker, regardless of the order cost of the supplier and the inventory management cost of the supplier, or it is a centralized decision-making that the supplier does not set up a distribution center. The task of the central decision-maker is to determine how the goods should be allocated among different demand points and how to distribute the goods to the demand point. The purpose of decision makers is to minimize the total cost of the whole secondary supply chain system, because the standard supply chain system is a typical R-System. Therefore, in the total cost, the supplier only considers the transportation cost in the process of distribution to the downstream retailer, while the retailer's cost includes the retailer's order cost, the retailer's inventory cost and the out-of-stock cost.

\subsubsection{Cost Analysis of Retailers}

(1) Inventory analysis of retailers

\section{1) Inventory Limit Analysis of Retailer $i$}

For retailers, if the inventory carrying capacity is greater, the frequency of suppliers replenishing goods to downstream retailers will decrease correspondingly in a certain period of time. If so, the cost of suppliers will undoubtedly be lower, but the problem is that retailers hold more goods because of the large inventory carrying capacity. In contrast, if the retailer's inventory cap is smaller, it will increase the frequency of suppliers replenishing retailers in a certain period of time, so that the retailer's inventory cost will be lower, but because replenishments to retailers are more frequent, suppliers will also replenish. Transportation costs are rising. Therefore, the inventory cap should be considered in the whole supply chain system, instead of making the inventory cap infinite. 


\section{2) Inventory Lower Limit Analysis of Retailer $i$}

Because retailers adopt $(t, R, S)$ as inventory strategy, which corresponds to $\left(t, L_{i}, U_{i}\right)$ inventory strategy established in this paper. In this inventory strategy, the key is to see the relationship between retailer's inventory level and $L_{i}$. If the inventory level of retailer $i$ is larger than that of retailer $L_{i}$ at the end of the $t$ cycle, then suppliers do not have to replenish the retailer.

Another way to understand this is that at the beginning of the $t+1$ cycle, retailer i's inventory should have all the lowest levels of $L_{i}+1$. If we require the retailer's customer service level not to be lower than $d_{i,}$ that is, $\mathrm{P}\left\{x_{(t+1) i}=L_{i}+1\right\} \geq d_{i}$, and because $X_{i}$ obeys the normal distribution $N$ $\left(\mu_{i}, \sigma_{i}^{\wedge} 2\right)$, then there is a minimum value for $L_{i}$.

$$
\varnothing\left(\frac{L_{i}+1-\mu_{i}}{\sigma_{i}}\right)=d_{i}
$$

For all retailers, assuming that their inventory level at the end of this cycle is greater than the minimum inventory level $L_{i}$, the initial inventory level of the retailer at the beginning of the next cycle is equal to the inventory level at the end of this cycle; on the contrary, assuming that their inventory level at the end of this cycle is less than the minimum inventory level $L_{i}$, then it is zero. The initial inventory of the seller in the next cycle will become the maximum after supplier replenishment. The formula can be expressed more directly as follows:

$$
\begin{gathered}
S_{(t+1) i}=\left\{\begin{array}{cc}
U i, & S_{t i}-x_{t i} \leq L_{i} \\
S_{t i}-x_{t i}, S_{t i}-x_{t i}>L_{i}
\end{array}\right. \\
S_{(t+1) i}=\left\{\begin{array}{cc}
U i, & S_{t i}-x_{t i} \leq L_{i} \\
S_{t i}-x_{t i}, S_{t i}-x_{t i}>L_{i}
\end{array}\right.
\end{gathered}
$$

\subsubsection{Retailer Cost Analysis}

Assuming that under the premise of no replenishment, the retailer's main expenses mainly include three, namely, the ordering cost, the inventory holding cost and the shortage cost.

\section{1) Order cost}

We set the order cost as $C O$. The order cost borne by the retailer mainly depends on the quantity of goods delivered by the supplier to the retailer and the instant price of perishable products when they arrive at the retailer. Because retailers adopt $(s, R, T)$ inventory strategy, the distribution volume of perishable products from suppliers to retailers varies according to the retailer's inventory. When the retailer is out of stock at the end of the period, the perishable products that the supplier distributes to the retailer are the upper limit of the retailer's inventory; when the retailer's final inventory is less than the lower limit of the inventory, the quantity of perishable products that the supplier distributes to the retailer is the difference between the upper limit of the retailer's inventory and the retailer's final inventory; when the retailer's final When the inventory is larger than its lower limit, the supplier 
does not need to distribute the goods to the retailer. The mathematical expression is as follows: the supplier's distribution to retailer $I$ at the end of the t-cycle is as follows:

$$
Q_{t i}=\left\{\begin{array}{cc}
U_{i}, & S_{t i}-x_{t i} \leq 0 \\
U_{i}-\left(S_{t i}-x_{t i}\right), & 0<S_{t i}-x_{t i}<L_{i} \\
0, & S_{t i}-x_{t i}>L_{i}
\end{array}\right.
$$

As for the freshness loss of perishable products, the freshness of perishable products is related to the transportation distance. The larger the transportation distance the greater the freshness loss. This will further affect the price of the product. Therefore, the focus of this paper is how to determine the loss of freshness. Assuming the distribution route of perishable products is $i$, it needs to be analyzed first. Suppose that the last retailer is retailer $g$ before the delivery vehicle arrives at retailer I and the last retailer on Retailer $\mathrm{G}$ is retailer $j$. In the $t$-cycle, zero-i vendor I is distributed by vehicle $M_{0}$, supplier-retailer $i$ is distributed by $l_{t i}$ and supplier-retailer $\mathrm{J}$ is distributed by $l_{t j}$.

$$
l_{t i}=l_{t j}+X_{M_{0} t j g} d_{j g}+X_{M_{0} t g i} d_{g i}+X_{M_{0} t j i} d_{j i}
$$

where $\left(X_{M_{0} t j g}+X_{M_{0} t g i}\right) X_{M_{0} t i j}=0$

Similarly, the same analogy is applied to the distribution distance of other retailers before the retailer $j$, and the final value of $l_{t j}$ can be obtained. At this point, the price of perishable products to retailer I can be determined by

$\psi_{t i}=\alpha\left(e^{b l_{t i}}-1\right)$

It can be determined that the price of perishable products to retailer $i$ is

$$
P_{t i}=P v \cdot\left(1-\Psi_{t i}\right)
$$

Then, retailer I's t-cycle order cost is:

$$
\begin{aligned}
& C O_{t i}=P_{t i} \cdot Q_{t i}= \\
& \left\{\begin{array}{lr}
P v \cdot\left(1-\psi_{t i}\right) U_{i}, & S_{t i}-x_{t i} \leq 0 \\
P v \cdot\left(1-\psi_{t i}\right)\left(U_{i}-\left(S_{t i}-x_{t i}\right)\right), & 0<S_{t i}-x_{t i}<L_{i} \\
0, & S_{t i}-x_{t i}>L_{i}
\end{array}\right.
\end{aligned}
$$

Therefore, the order cost of all retailers is

$$
\mathrm{CO}=\sum_{t=1}^{m} \sum_{i=1}^{n} C O_{t i}
$$




\section{2) Inventory Cost of Retailer I in the Third Cycle}

Inventory cost of retailer is $I C$. In order to express it more directly and clearly, this paper will take the average inventory of each cycle as the research object, that is, to sum and average the initial inventory level and the end inventory level of a certain cycle.

When there is $s_{t i}-x_{t i}<=0$ and the inventory at the end of the period is zero, the average inventory in the T period is $s_{t i} / 2$.

When there is $0<s_{t i}-x_{t i}<=\mathrm{Li}$ and the inventory at the end of the period is not zero, the average inventory in the T period is $\left(2 s_{t i}-x_{t i}\right) / 2$.

When there is $s_{t i}-x_{t i}>\mathrm{Li}$, that is, when there is no need to replenish the retailer, the average inventory in the T period is $\left(2 s_{t i} x_{t i}\right) / 2$.

Then retailer $i$ 's $t$-cycle inventory cost is:

$$
I C_{t i}=\left\{\begin{array}{cc}
\ln \left(h_{i} /(1+m \mu)\right) \cdot S_{t i} / 2 & S_{t i}-x_{t i} \leq 0 \\
\ln \left(h_{i} /(1+m \mu)\right) \cdot\left(2 S_{t i}-x_{t i}\right) / 2 & 0<S_{t i}-x_{t i}<L_{i} \\
\ln \left(h_{i} /(1+m \mu)\right) \cdot\left(2 S_{t i}-x_{t i}\right) / 2 & S_{t i}-x_{t i}>L_{i}
\end{array}\right.
$$

Therefore, the inventory cost of all retailers is $\mathrm{IC}=\sum_{t=1}^{m} \sum_{i=1}^{n} I C_{t i}$.

\section{3) Shortage Cost of Retailer I in the Third Cycle}

Suppose the cost of shortage of retailers is $S C$. We Know that if the demand of consumers is greater than the inventory level of perishable products of retailers at the beginning of this cycle, there will be a shortage of supply and demand, that is, shortage. At this time, the shortage of retailer I in the t-cycle is $x_{t i}-s_{t i}$, and the cost of shortage is

$$
S C_{t i}=p \cdot\left(X_{t i}-S_{t i}\right)
$$

Therefore, the shortage cost of all retailers is $\mathrm{SC}=\sum_{t=1}^{m} \sum_{i=1}^{n} S C_{t i}$.

When the retailer's inventory at the end of the period is larger than its inventory limit, $0<=x_{t i}<s_{t i}$ $L_{i}$, the supplier is not required to distribute the goods to the retailer and there is no shortage of the goods, so the retailer's cost at this time only includes the inventory cost.

$$
\begin{aligned}
& C_{1}=\sum_{t=1}^{m} \sum_{i=1}^{n} S C_{t i} \\
& =\sum_{t=1}^{m} \sum_{i=1}^{n} \ln \left(h_{i} \cdot\left(2 S_{t i}-x_{t i}\right) / 2\right)
\end{aligned}
$$

When the retailer's final inventory is greater than zero but less than the retailer's lower inventory limit, i.e. $s_{t i} L_{i}<=x_{t i}<s_{t i}$, the supplier is required to replenish the inventory. At this time, the retailer's cost includes the order cost and inventory cost. 


$$
\begin{aligned}
& C_{1}=\sum_{t=1}^{m} \sum_{i=1}^{n} C O_{t i}+\sum_{t=1}^{m} \sum_{i=1}^{n} S C_{t i} \\
& =\sum_{t=1}^{m} \sum_{i=1}^{n} \operatorname{PV} \cdot\left(1-\psi_{t i}\right)\left(\mu_{i}-\left(S_{t i}-x_{t i}\right)\right) \\
& +\sum_{t=1}^{m} \sum_{i=1}^{n} h_{i} \cdot\left(2 S_{t i}-x_{t i}\right) / 2
\end{aligned}
$$

When the retailer's inventory at the end of the period is out of stock, that is, $x_{t i}>s_{t i}$, the retailer's cost includes not only the order cost and inventory cost, but also the out-of-stock cost.

$$
\begin{aligned}
& C_{1}=\sum_{t=1}^{m} \sum_{i=1}^{n}\left(C O_{t i}+I C_{t i}+S C_{t i}\right) \\
& =\sum_{t=1}^{m} \sum_{i=1}^{n} \operatorname{Pv} \cdot\left(1-\gamma_{t i}\right) \mu_{i} \\
& +\sum_{t=1}^{m} \sum_{i=1}^{n} h_{i} \cdot \frac{S_{t i}}{2}+\sum_{t=1}^{m} \sum_{i=1}^{n} p\left(x_{t i}-S_{t i}\right)
\end{aligned}
$$

\subsubsection{Supplier Cost Analysis}

Because this paper does not consider the inventory cost of suppliers, the cost of suppliers is mainly in the process of distribution of goods.

Transportation costs incurred. In this paper, the transportation cost is mainly divided into two components: the fixed starting cost and the fixed starting cost of the vehicle.

The total transportation cost is:

$$
C_{2}=m c_{0}+c_{1} \cdot \sum_{k=1}^{K} \sum_{t=1}^{m} \sum_{i=0}^{n} \sum_{j=0}^{n} X_{k t i j} d_{i j}
$$

\subsection{Model Establishment}

Through the above analysis, the total cost of the system is divided into four parts: retailer's order cost, retailer's inventory holding cost, retailer's shortage cost and supplier's transportation cost. However, under different demands, there will be different total cost calculation. When $x_{i}>=\& \& x_{i}<s_{t i}-L_{i}$ :

$$
\begin{aligned}
& \mathrm{TC}=C_{1}+C_{2}=\sum_{t=1}^{m} \sum_{i=1}^{n} h_{i} \cdot \frac{\left(2 S_{t i}-x_{t i}\right)}{2}+m c_{0} \\
& +c_{1} \cdot \sum_{M=1}^{M} \sum_{t=1}^{m} \sum_{i=0}^{n} \sum_{j=0}^{n} X_{M t i j} d_{i j}
\end{aligned}
$$

When $x_{i}<=s_{t i} \& \& x_{i}>=s_{t i}-L_{i}$ 


$$
\begin{aligned}
& \mathrm{TC}=C_{1}+C_{2}=\sum_{t=1}^{m} \sum_{i=1}^{n} \mathrm{PV} \cdot\left(1-{ }_{t i}\right)\left(\mu_{i}-\left(S_{t i}-x_{t i}\right)\right) \\
& +\sum_{t=1}^{m} \sum_{i=1}^{n} h_{i} \cdot \frac{\left(2 S_{t i}-x_{t i}\right)}{2}+m c_{0} \\
& +c_{1} \cdot \sum_{k=1}^{K} \sum_{t=1}^{m} \sum_{i=0}^{n} \sum_{j=0}^{n} X_{k t i j} d_{i j}
\end{aligned}
$$

When $x_{i}>s_{t i}$

$$
\begin{aligned}
& \mathrm{TC}=C_{1}+C_{2}=\sum_{t=1}^{m} \sum_{i=1}^{n} \mathrm{Pv} \cdot\left(1-\gamma_{t i}\right) \mu_{i} \\
& +\sum_{t=1}^{m} \sum_{i=1}^{n} h \cdot \frac{\left(S_{t i}\right)}{2}+\sum_{t=1}^{m} \sum_{i=1}^{n} p\left(x_{t i}-S_{t i}\right)+m c_{0} \\
& +c_{1} \cdot \sum_{M=1}^{M} \sum_{t=1}^{m} \sum_{i=0}^{n} \sum_{j=0}^{n} X_{M t i j} d_{i j}
\end{aligned}
$$

\subsection{Model Conversion}

In practical problems, because the demand of perishable products $X_{t i}$ obeys the random variable of normal distribution $N\left(\mu_{i}, \sigma_{i}^{\wedge} 2\right)$, the whole function TC contains random variable, and then the objective function also contains random variable, which makes it impossible to find the minimum value of the desired objective function and can not be directly transported through the model. Calculate and solve. We use the stochastic expectation model to determine the stochastic demand, transform the stochastic variable into the deterministic variable, and then further solve the objective function. So the ultimate model to minimize the total cost of the system is:

$$
\begin{aligned}
& T C^{\prime}=C_{1}+C_{2}=\sum_{t=1}^{m} \sum_{i=1}^{n} \int_{0}^{S_{t i}-L_{i}} \frac{\operatorname{lnh} h_{i}\left(2 S_{t i}-x_{t i}\right)}{2 d F(x)} \\
& +\sum_{t=1}^{m} \sum_{i=1}^{n} \int_{S_{t i}-L_{t i}}^{S_{t i}} \operatorname{Pv} \cdot\left(1-{ }_{t i}\right)\left(\mu_{i}-\left(S_{t i}-x_{t i}\right)\right) d F(x) \\
& +\sum_{t=1}^{m} \sum_{i=1}^{n} \int_{S_{t i}-L_{t i}}^{S_{t i}} h_{i} \cdot \frac{\left(2 S_{t i}-x_{t i}\right)}{2} d F(x) \\
& +\sum_{t=1}^{m} \sum_{i=1}^{n} \int_{S_{t i}}^{+\infty} \operatorname{Pv} \cdot\left(1-\gamma_{t i}\right) \mu_{i} d F(x) \\
& +\sum_{t=1}^{m} \sum_{i=1}^{n} \frac{h_{i} S_{t i}}{2 d F(x)}+\sum_{t=1}^{m} \sum_{i=1}^{n} p\left(x_{t i}-S_{t i}\right) \\
& +m c_{0}+c_{1} \cdot \sum_{M=1}^{m} \sum_{t=1}^{s} \sum_{i=0}^{n} \sum_{j=0}^{n} X_{M t i j} d_{i j}
\end{aligned}
$$




$$
\begin{aligned}
& \text { s.t. } \sum_{i=1}^{n} Q_{t i} Y_{k t i} \leq W, \forall k \in K, t \in T \\
& 0 \leq L_{i} \leq U_{i} \leq Q_{i}^{\prime}, \forall i \in N \\
& \sum_{M=1}^{m} Y_{k t i}=1, \quad \forall t \in T, i \in N \\
& \sum_{i=0}^{N} x_{k t o i}=\sum_{j=0}^{N} x_{k t o j} \leq 1, \quad \forall k \in K t \in T \\
& \sum_{k=1}^{K} \sum_{\substack{i=0 \\
i \neq j}}^{n} X_{k t i j}=1 \forall i \in N, j \in N, t \in T \\
& \sum_{i=0}^{n} X_{M t i j}=Y_{M t j}, \quad \forall j \in N, M \in m, t \in T \\
& \sum_{j=0}^{n} X_{k t i j}=Y_{k t i}, \quad \forall i \in N, M \in m, t \in T \\
& X_{M t i j} \in\{0,1\}, \forall i, j \in N, M \in m, t \in T
\end{aligned}
$$

In the model constructed above, constraint (23) indicates that the quantity of goods transported by a vehicle does not exceed its maximum load capacity; constraint (24) indicates that the retailer's inventory limit and inventory limit should be between 0 and the maximum inventory; constraint (25) ensures that each demand point has one and only one vehicle. Vehicle serves it, each demand point is only on one path and does not go through a loop; constraint (26) means that the starting point and final destination of each vehicle are suppliers; constraint (27) means that when two retailers appear on a distribution path, they must replenish their goods; (28) and (29) means that, for example, when two retailers appear on a distribution path, they must replenish their goods. If the retailer needs to distribute, then when the vehicle $\mathrm{M}$ passes through the retailer, it must serve the retailer; (30) the variable is $0-1$. 


\section{ALGORITHMIC DESIGN}

\subsection{Basic Ideas of Differential Evolution Algorithms}

Assuming that the problem to be solved is a minimization problem, the mathematical model of the problem is $\min f\left(x_{1}, x_{2}, \ldots, x_{n}\right)$, where $x_{j} \in\left[L_{j}, U_{j}\right]$, and $1<=j<=$ n. $X(0)$ is the initial population, so $X_{\mathrm{i}}(t)=\left(x_{\mathrm{i} 1}(t), x_{\mathrm{i} 2}(t), \ldots, x_{\mathrm{in}}(t)\right)$ is the first individual $i \mathrm{n}$ the t-generation population, the population is n-dimensional spatial structure, and the population size is $N P$. Let $\mathrm{X} 1$ and $\mathrm{X} 2$ be two different individuals, then the difference vector formed by them is $X_{1}-X_{2}$.

The differential evolution algorithm is described as follows:

Step 1 randomly generates the initial population:

$P(0)=\left\{X_{\mathrm{i}}(0) \mid x_{\mathrm{ij}}(0)=\operatorname{rand}(0,1) *\left(U_{j}-L_{j}\right)+L_{j,} 1 \leq i \leq \mathrm{s} \cap 1 \leq j \leq \mathrm{n}\right\}$

The Rand $(0,1)$ function is to find the random number on $(0,1)$ and set the initial value of the evolution time $\mathrm{t}=0$.

Step 2: Perform mutation operation: Randomly select three different individuals $X_{a}(t), X_{b}(t$, $X_{c}(t), \mathrm{a} \neq \mathrm{b} \neq \mathrm{c}, \mathrm{a} \_\mathrm{b} \_\mathrm{c}$ from the current population $\mathrm{P}(\mathrm{t})$. Calculate individual differences $D_{i}(t+1)=\left(d_{i 1}(t+1), d_{i 2}(t+1), \ldots, d_{i n}(t+1)\right)$ :

$d_{i j}(t+1)=x_{a j}(t)+F *\left(x_{b j}(t)-x_{c j}(t)\right)$

Where $1<i<\mathrm{s}, 1<j<\mathrm{n}$;

Step 3 performs the crossover operation: randomly generate the random decimal $R_{1}<(0,1)$ and the random integer $R_{2}<[1, \mathrm{n}]$, and calculate the temporary individual $E_{\mathrm{i}}(t+1)=\left(e_{i 1}(t+1), e_{i 2}(t+1), \ldots\right.$, $\left.e_{i n}(t+1)\right)$ :

$e_{i j}(t+1)=\left\{\begin{array}{cc}d_{i j}(t+1) & r_{1} \leq \text { CRor } r_{2}=j \\ x_{i j}(t) & \text { otherwise }\end{array}\right.$

Where $1<i<\mathrm{s}$ and $1<j<\mathrm{n}$;

Step 4 performs the selection operation:

$x_{i}(t+1)=\left\{\begin{array}{cc}e_{i}(t+1) & \text { if } f\left(e_{i}(t+1)\right)<f\left(x_{i}(t)\right) \\ x_{i}(t) & \text { otherwise }\end{array}\right.$

Where $1<i<\mathrm{s}$;

Step 5 calculates $X_{\text {best }}(t+1)$ of the individuals with the smallest fitness in population $P(t+1)$.

Step 6 If the termination condition is not satisfied, then $\mathrm{t}=\mathrm{t}+1$ and step 2; otherwise output $X_{\text {best }}$ and $\mathrm{f}\left(X_{\text {best }}\right)$, and end. 


\subsection{Improvement of Differential Evolution Algorithms}

As the number of retailers increases, the running time of the algorithm will increase exponentially, and the accuracy of the algorithm will decrease. In order to accelerate the convergence speed and improve the accuracy of the algorithm, the classical differential evolution algorithm must be improved.

In order to accelerate the convergence speed of the algorithm, the following measures are taken:

When the initial population is initialized, the traditional idea of random generation is changed, and the grid thinking is adopted to distribute the individual of the initial population evenly into the solution space. For customer I inventory, $\left[L_{j}, U_{j}\right]$ can be divided into n intervals.

$P(0)=\left\{X_{\mathrm{i}}(0) \mid x_{\mathrm{ij}}(0)=\mathrm{i}^{*}\left(U_{j}-L_{j}\right) / \mathrm{n}+L_{j,}\{1<\mathrm{i}<\mathrm{s}, 1<\mathrm{j}<\mathrm{n}\}\right.$

On the basis of step 4 of Section 3.1, greedy algorithm is introduced to find the optimal solution based on $\mathrm{Xi}(\mathrm{t})$. The algorithm is as follows:

While $n m<N m$

If $f\left(x_{i}\right)>f\left(x_{i}+\sin \left(p_{i} / 2 * n m\right)\right)$ then $x_{i}=x_{i}+\sin \left(\left(p_{i} / 2\right) * n m\right)$;

$N m=n m+1$

End while

In order to improve the accuracy of the algorithm, some methods are adopted to avoid falling into local optimum. The specific measures are as follows:

The scaling factor $\mathrm{F}$ of the mutation operation of the classical differential evolution algorithm is adaptively and dynamically changed. The value of the scaling factor decreases as the evolution algebra increases, $F=1+e^{-n}$

Secondly, two evolutionary models, DE/rand/1/bin and DE/rand-to-best/1/bin, were chosen randomly, or two populations were used to adopt different evolutionary models. Because DE/rand/1/ bin evolutionary model has high precision, but the convergence speed of the algorithm is relatively slow, while DE/rand-to-best/1/bin evolutionary model has the opposite speed, but it is easy to fall into local optimum.

For the condition of selection operation, aiming at the IRP problem, all constraints are neglected selectively, and the f'value is obtained. That is to change the fitness function appropriately. The choice here is conditional. We use $f$ as the fitness value on the premise that the population tends to concentrate and is in danger of falling into the local optimum. Here we use the variance of the population to judge the degree of population aggregation. When the variance is less than a threshold, we use the cancellation of all constraints to calculate the fitness value.

\section{SIMULATION EXPERIMENT}

Suppose there is a one-to-many two-stage perishable product distribution system, in which there are one supplier and 20 retailers. The system plan period is 7 days, and every day counts as a cycle. Delivery of perishable products is apple, freshness loss function $\Psi=a e^{k l}-a$, which is determined by analysis that $\mathrm{a}=0.05, \mathrm{~B}=0.003$. The fixed start-up cost of suppliers delivering goods to retailers is 150 yuan per day, the transportation cost of vehicles is 2 yuan/Km, the maximum load of transport vehicles is 900 pieces, the unit inventory cost of retailers is 1 yuan/piece per day, the delivery price of perishable products from suppliers is 40 yuan/piece, and the unit out-of-stock cost of retailers is 4 yuan/ piece. The retailer's maximum inventory is 300 pieces. Retailers have the same level of service. The numbers of suppliers and retailers and the corresponding left-hand parameters are shown in Table 1.

This paper establishes a joint optimization model for inventory and transportation of perishable products under Stochastic Demand Based on vendor managed inventory (VMI) with seven- 
day planning period. The objective function is the total cost of the system, which includes four parts: retailer's order cost, retailer's inventory holding cost, retailer's shortage cost and supplier's transportation cost. In this paper, the improved differential evolution algorithm is used to solve the mathematical model, and the output results are obtained (Figure 1).

The whole distribution route of IDE algorithm is:

First day:

$0-10(276)-3(278)-7(288)-0-6(245)-4(230)-2(227)-$

$0-19(201)-14(247)-9(211)-0-12(203)-16(231)-20(274)-$

$0-15(209)-17(235)-0$

The second day:

$0-8(261)-18(254)-1(142)-5(222)-0$

The third day:

$0-3(270)-19(180)-9(180)-0$

The fourth day:

$0-3(261)-11(180)-7(210)-5(210)-$

$0-6(180)-4(240)-14(210)-15(240)-0-8(284)-10(290)-0$

The fifth day:

$0-19(180)-9(180)-0-12(240)-17(240)-16(280)-20(240)-0$

The sixth day:

Table 1. Relevant parameters of suppliers and retailers

\begin{tabular}{|c|c|c|c|}
\hline Retailer Number & coordinate & Initial inventory & $\left(\boldsymbol{\mu}, \boldsymbol{\sigma}^{2}\right)$ \\
\hline 0 & 50,50 & & \\
\hline 1 & 23,80 & 100 & 50,400 \\
\hline 2 & 31,23 & 50 & 50,900 \\
\hline 3 & 22,85 & 0 & 70,400 \\
\hline 4 & 28,66 & 33 & 80,400 \\
\hline 5 & 3,98 & 258 & 60,1600 \\
\hline 6 & 34,61 & 25 & 80,4900 \\
\hline 7 & 4,84 & 0 & 50,900 \\
\hline 8 & 48,38 & 159 & 60,400 \\
\hline 9 & 5,24 & 68 & 90,900 \\
\hline 10 & 65,63 & 0 & 70,400 \\
\hline 11 & 7,72 & 239 & 50,900 \\
\hline 12 & 72,28 & 87 & 60,900 \\
\hline 13 & 53,59 & 150 & 50,400 \\
\hline 14 & 32,41 & 123 & 60,1600 \\
\hline 15 & 78,32 & 76 & 80,900 \\
\hline 16 & 90,12 & 68 & 50,400 \\
\hline 17 & 96,16 & 135 & 70,100 \\
\hline 18 & 65,61 & 226 & 90,1600 \\
\hline 19 & 48,33 & 90 & 70,2500 \\
\hline 20 & 83,3 & 86 & 50,1600 \\
\hline
\end{tabular}


Figure 1. inventory routing optimization total cost

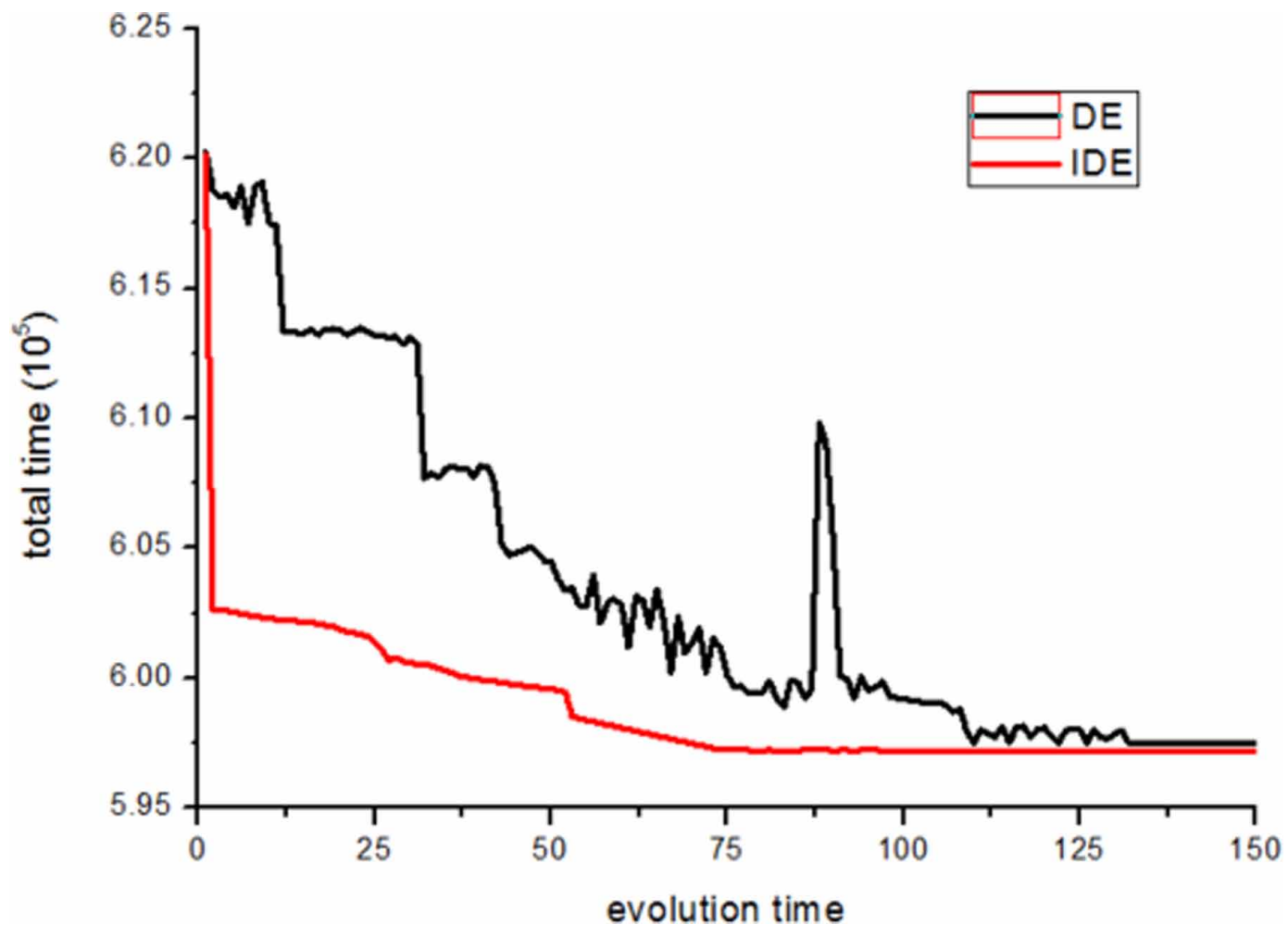

$0-5(180)-1(180)-14(240)-8(240)-0-18(253)-0$

The seventh day:

$0-6(240)-4(240)-7(240)-3(210)-0-9(180)-$

$0-14(240)-19(240)-10(180)-15(210)-0$

The whole distribution route of $\mathrm{DE}$ algorithm is:

The firth day:

$0-3(292)-7(270)-10(254)-0-6(255)-4(242)-$

$0-2(200)-19(211)-14(187)-9(207)-$

$0-20(211)-15(239)-17(256)-0-12(201)-16(274)-0$

The second day:

$0-8(272)-18(209)-1(176)-5(203)-0$

The third day:

$0-3(270)-19(180)-9(180)-0$

The fourth day:

$0-11(180)-7(210)-5(210)-0-6(180)-4(240)-$

$14(210)-15(240)-0-8(284)-10(290)-0$

The fifth day:

$0-3(230)-19(180)-9(180)-$

$0-12(240)-17(240)-16(280)-20(240)-0$

The sixth day:

$0-8(240)-14(180)-1(240)-5(180)-0-18(250)-0$

The seventh day: 
Figure 2. Order value

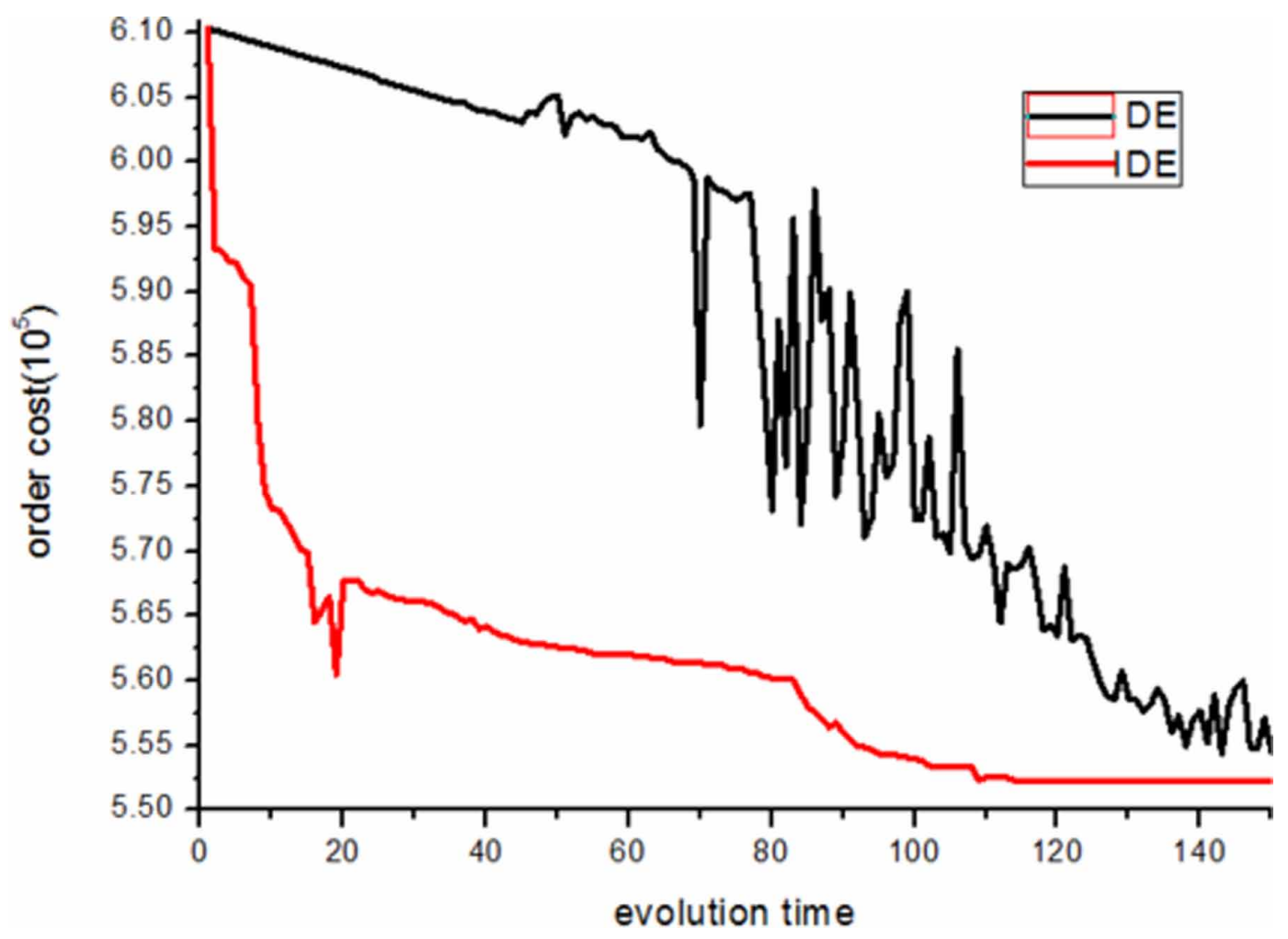

$0-3(240)-7(240)-4(240)-6(210)-0-9(180)-$

$0-15(240)-18(240)-19(180)-14(211)-0$

After IDE algorithm optimization, the total cost of the secondary supply chain is 483577 yuan, of which the retailer's order cost is 455240 yuan, the retailer's inventory cost is 23207 yuan, the retailer's out-of-stock cost is 365 yuan, and the supplier's transportation cost is 4765 yuan. The transportation distance is 2,031.72 Milometers

After optimization by DE algorithm, the total cost of the secondary supply chain is 493139 yuan, of which the retailer's order cost is 463018 yuan, the retailer's inventory cost is 24582 yuan, the retailer's out-of-stock cost is 407 yuan, and the supplier's transportation cost is 5132 yuan. The transportation distance is 2,408.03 Milometers

The numerical experiments show that the IDE algorithm proposed in this paper is not only feasible in solving the IRP problem of perishable products, but also has great superiority over DE algorithm Figure 3).

\section{SUMMARY}

Inventory and distribution of perishable products is a very difficult problem, because it is an IRP problem and an N-P problem. In addition, perishable products have their own particularities. With the extension of distribution time and inventory time, their value will gradually decrease. In order to arrange the distribution time, quantity and route of each customer point reasonably, on the basis of some assumptions, taking inventory and vehicle as constraints, and aiming at optimizing the total cost of the system, a mathematical model is established. Aiming at the particularity of perishable goods 
Figure 3. Routing cost

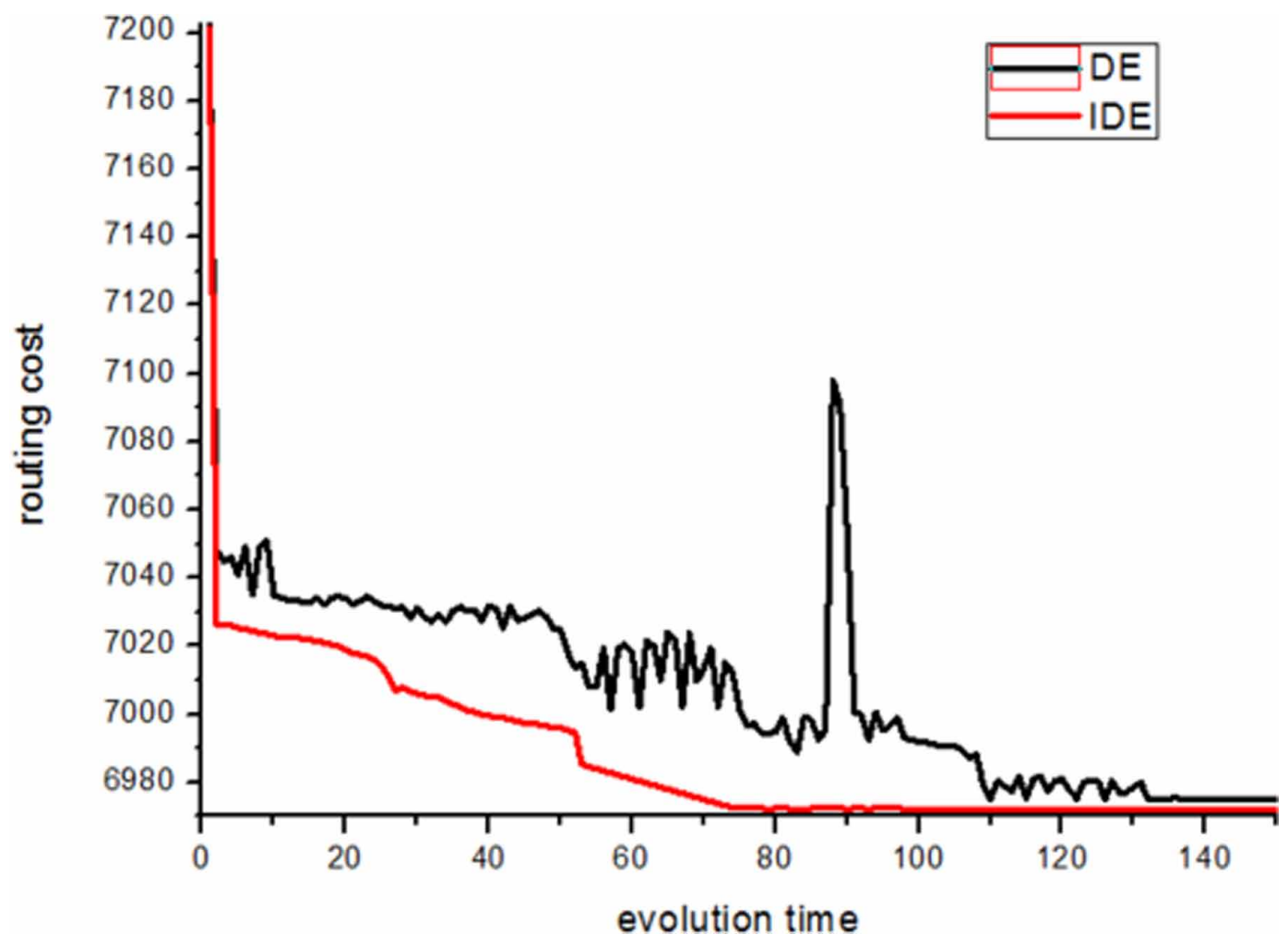

inventory routing problem, the differential evolution algorithm is presented from two perspectives. The degree of convergence is improved by grid initialization and greedy local optimization algorithm combined with differential evolution algorithm. The accuracy of the algorithm is improved by adaptive scaling factor, two evolution modes and changing the constraints of the problem. Then the improved algorithm is used to solve the inventory routing problem. The numerical results show that the algorithm is effective and feasible, and the performance of the algorithm is greatly improved compared with the DE algorithm. It can be seen from Figure 2 that when the algorithm evolves to the 20th generation, it is found that the solution of a certain process deteriorates obviously, which slows down the convergence speed of the algorithm. In the future, we should further study how to improve the convergence speed of the algorithm. 


\section{REFERENCES}

Alvarez, A., Cordeau, J.-F., Jans, R., Munari, P., \& Morabito, R. (2020). Formulations, branch-and-cut and a hybrid heuristic algorithm for an inventory routing problem with perishable products. European Journal of Perational Research, 2(6), 511-529. doi:10.1016/j.ejor.2019.11.015

Alvareza,, A., Jean-Franc, O. C., \& Jansb, R. (2019). Formulations, Branch-and-Cut and a Hybrid Heuristic Algorithm for an Inventory Routing Problem with Perishable Products. European Journal of Operational Research.

Bhattacharjee, S., \& Ramesh, R. (2007). A multi-period profit maximizing model for retail supply chain management:An integration of demand and supply-side mechanisms. European Journal of Operational Research, 122(3), 584-601. doi:10.1016/S0377-2217(99)00097-1

Darestani, S. A., \& Mousazadeh, S. (2019). Modeling a production-inventory-routing problem of blood products using heuristic solution methods. Journal of Intelligent \& Fuzzy Systems, 37(1), 1-21.

Devapriya, P., Ferrell, W., \& Geismar, N. (2017). Integrated production and distribution scheduling with a perishable product. European Journal of Operational Research, 259(3), 906-916. doi:10.1016/j.ejor.2016.09.019

Donselaar, M. V., Woensel, T. V., \& Broekmeulen, R. (2006). Inventory control of perishables in supermarkets. International Journal of Production Economics, 104(2), 462-472. doi:10.1016/j.jpe.2004.10.019

Goyal, S. M., \& Giri, B. C. (2001). Recent trends in modeling deteriorating inventory. European Journal of Operational Research, 134(1), 1-16. doi:10.1016/S0377-2217(00)00248-4

Herbon, A., \& Khmelnitsky, E. (2017). Optimal dynamic pricing and ordering of a perishable product under additive effects of price and time on demand. European Journal of Operational Research, 60(2), 546-556. doi:10.1016/j.ejor.2016.12.033

Jafarkhan, F., \& Yaghoubi, S. (2018). An efficient solution method for the flexible and robust inventory-routing of red blood cells. Computers \& Industrial Engineering, 117, 191-206. doi:10.1016/j.cie.2018.01.029

Levin, Y., McGill, J., \& Nediak, M. (2008). RisM in revenue management and dynamic pricing. Operations Research, 56(2), 326-343. doi:10.1287/opre.1070.0438

Li, K., Chen, Y., Li, W., He, J., \& Xue, Y. (2018). Improved gene expression programming to solve the inverse problem for ordinary differential equations. Swarm and Evolutionary Computation, 38, 231-239. doi:10.1016/j. swevo.2017.07.005

Li, K., Liang, Z., Yang, S., Chen, Z., Wang, H., \& Lin, Z. (2019). Performance Analyses of Differential Evolution Algorithm Based on Dynamic Fitness Landscape. International Journal of Cognitive Informatics and Natural Intelligence, 13(1), 36-61. doi:10.4018/IJCINI.2019010104

Li, K., Wang, H., \& Li, S. (2019). A mobile node localization algorithm based on an overlapping self-adjustment mechanism. Information Sciences, 481, 635-649. doi:10.1016/j.ins.2018.12.006

Li, Y., Zhang, S., \& Han, J. (2017). Dynamic Pricing and periodic ordering for a stochastic inventory system with deteriotatong items. Automatica, 5, 200-213. doi:10.1016/j.automatica.2016.11.003

Ming \& Li. (2018). Cost optimization problem with soft time window for fresh food distribution based on improved fuzzy genetic algorithm. Mathematical Problems in Engineering.

Mirzaei, S., \& Seifi, A. (2015). Considering Lostsale in inventory routing problems for perishable goods. Computers \& Industrial Engineering, 47, 213-227. doi:10.1016/j.cie.2015.05.010

Mjirda, A., Jarboui, B., Macedo, R., Hanafi, S., \& Mladenović, N. (2014). A two phase variable neighborhood search for the multi-product inventory routing problem. Computers \& Operations Research, 52, 291-299. doi:10.1016/j.cor.2013.06.006

Rafie-Majd, Z., Pasandideh, S. H. R., \& Naderi, B. (2017). Modelling and Solving the Integrated InventoryLocation-Routing Problem in a multi-period and multi-perishable Product Supply Chain with Uncertainty: Lagrangian Relaxation Algorithm. Computers \& Chemical Engineering, 21, 56-67. 
Wang, F., Li, Y., Zhang, H., Hu, T., \& Shen, X. (2019). An adaptive weight vector guided evolutionary algorithm for preference-based multi-objective optimization. Swarm and Evolutionary Computation, 49, 220-233. doi:10.1016/j.swevo.2019.06.009

Wang, F., Zhang, H., Li, K., Lin, Z., Yang, J., \& Shen, X.-L. (2018). A Hybrid Particle Swarm Optimization Algorithm Using Adaptive Learning Strategy. Information Sciences, 436-437, 162-177. doi:10.1016/j. ins.2018.01.027

Wouter, L., El-Houssaine, A., \& Khaled, H. H. (2018). Analysis of an improved branch-and-cut formulation for the Inventory-Routing Problem with Transshipment. Computers \& Operations Research, 98, 137-148. doi:10.1016/j.cor.2018.05.023

Zhang, Miao, \& Guan. (2018). Distribution path optimization of fresh agricultural products under O2O model. Logistics Technology, 37(12), 78-82, 88.

Zelin Wang is an Associate Professor at school of information science and technology, Nantong University. He received his PhD in 2015. His research focuses on research and application of intelligent algorithms.

Xiaoning Wei is an Associate Professor at school of information Science and Technology, Nantong University. He received his Master in School of Computer Science and Technology, Suzhou University, His research focuses on research and application of intelligent algorithms.

Jiansheng Pan is a lecturer at school of information Science and Technology, Nantong University. He received his Master in School of Computer Science and Technology, Suzhou University, His research focuses on research and application of intelligent algorithm. 